\title{
WD40 Repeat Protein 26 Negatively Regulates Formyl Peptide Receptor-1 Mediated Wound Healing in Intestinal Epithelial Cells
}

\author{
Mizuho Hasegawa, Charles A. Parkos, and Asma Nusrat
}

From the Department of Pathology, University of Michigan, Ann Arbor, Michigan

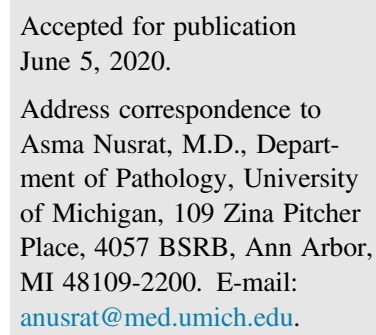

\begin{abstract}
$\mathrm{N}$-formyl peptide receptors (FPRs) serve as phagocyte pattern-recognition receptors that play a crucial role in the regulation of host defense against infection. Epithelial cells also express FPRs, and their activation during inflammation or injury results in enhanced epithelial migration and proliferation and improved mucosal wound repair. However, signaling mechanisms that govern epithelial FPR1 activity are not well understood. This study identified a novel FPR1-interacting protein, WD40 repeat protein (WDR)-26, which negatively regulates FPR1-mediated wound healing in intestinal epithelial cells. We show that WDR26-mediated inhibition of wound repair is mediated through the inhibition of Rac family small GTPase 1 and cell division cycle 42 activation, as well as downstream intracellular reactive oxygen species production. Furthermore, on FPR1 activation with $\mathrm{N}$-formyl-methionyl-leucyl phenylalanine, WDR26 dissociates from FPR1, resulting in the activation of downstream cell division cycle 42/Rac family small GTPase 1 signaling, increased epithelial cell migration, and mucosal wound repair. These findings elucidate a novel regulatory function of WDR26 in FPR1-mediated wound healing in intestinal epithelial cells. (Am J Pathol 2020, 190: 2029-2038; https://doi.org/10.1016/j.ajpath.2020.06.005)
\end{abstract}

The innate immune response is the first line of host defense against pathogenic infection. Early recognition of pathogens and activation of host-protective mechanisms are achieved through pattern-recognition receptors that detect pathogen-associated molecular-pattern molecules and damage-associated molecular patterns. ${ }^{1,2}$ Formyl peptide receptors (FPRs) represent a class of pattern-recognition receptors that belong to a family of seven transmembrane G-protein-coupled receptors. Humans express three functional FPRs: FPR1, -2, and -3 , which are highly expressed in neutrophils and monocytes. ${ }^{3-5}$ In addition to myeloid cells, the expression of FPR 1 in intestinal epithelial cells (IECs) has been previously identified. ${ }^{6,7}$ FPRs recognize a variety of ligands, derived from both pathogens and host cells. An important and wellcharacterized ligand for FPR1 is the short formylated bacterial peptide $N$-formyl-methionyl-leucyl phenylalanine (fMLP). ${ }^{8,9}$ The activation of FPRs in host cells leads to the dissociation of FPRs from heterotrimeric G-proteins $(\mathrm{G} \alpha$ and $G \beta \gamma$ ), resulting in the activation of downstream signaling cascades that promote a number of host-defense and antimicrobial-immune responses. ${ }^{3-5}$

The intestinal epithelium functions as a dynamic, permeable barrier allowing for selective absorption of nutrients, while restricting access of luminal pathogens to underlying tissues. Several pathologic disease states (including inflammatory bowel disease) are characterized by mucosal tissue damage, wounds, and compromised epithelial barrier functioning. ${ }^{10-13}$

Efficient mucosal barrier repair in the gut requires coordinated collective IEC migration and proliferation. In the terminal phases of wound repair, epithelial cells stop migrating as the barrier function is restored. These reparative responses are influenced by endogenous mediators, as well as by bacterial products. ${ }^{14-18}$ It has been previously shown that fMLP and the endogenous pro-resolving

Supported by NIH grants DK089763 (A.N.), DK055679 (A.N.), DK079392 (C.P.), DK72564 (C.P.), and DK61379 (C.P.).

Disclosures: None declared. 
mediator annexin 1 promote intestinal epithelial repair through the activation of epithelial FPR signaling. ${ }^{7,19}$ These studies identified Rac family small GTPase 1 (Rac1)- and NADPH oxidase (NOX)-1-mediated activation of reactive oxygen species (ROS) signaling as being crucial downstream effectors of FPR1 activation. ${ }^{19}$ However, mechanisms that govern upstream FPR1 regulation remain less well defined.

This study identified a novel FPR1-interacting protein, WD40 repeat protein (WDR)-26, which was associated with negative regulation of FPR1-mediated wound healing in IECs. This inhibition of wound repair was apparently mediated through reduced Rac1 and cell division cycle 42 (CDC42) activation, as well as decreased intracellular ROS generation. Furthermore, WDR26 dissociated from FPR1 on stimulation of IECs with fMLP, suggesting that WDR26-FPR1 interactions represent an important stop signal for epithelial cell migration.

\section{Materials and Methods}

\section{Cell Culture and Establishment of Stable Cell Lines}

SKCO15 human IECs and HEK293T cells were grown in high-glucose (4.5 g/L) Dulbecco's modified Eagle's medium supplemented with $10 \%$ fetal bovine serum, 100 $\mathrm{U} / \mathrm{mL}$ penicillin, $100 \mu \mathrm{g} / \mathrm{mL}$ streptomycin, $15 \mathrm{mmol} / \mathrm{L}$ HEPES (pH 7.4), $2 \mathrm{mmol} / \mathrm{L}$ L-glutamine, and $1 \%$ nonessential amino acids on tissue culture-treated plastic. HT29/B6 IECs were grown in RPMI 1640 supplemented with $10 \%$ fetal bovine serum, $1 \% 100 \mathrm{U} / \mathrm{mL}$ penicillin, 100 $\mu \mathrm{g} / \mathrm{mL}$ streptomycin, and $2 \mathrm{mmol} / \mathrm{L}$ L-glutamine. T84 IECs were grown in a 1:1 mixture of Dulbecco's modified Eagle's medium and Ham's F12 medium supplemented with $10 \%$ fetal bovine serum, $1 \% 100 \mathrm{U} / \mathrm{mL}$ penicillin, $100 \mu \mathrm{g} / \mathrm{mL}$ streptomycin, $10 \mathrm{mmol} / \mathrm{L}$ HEPES (pH 7.5), and $14 \mathrm{mmol} / \mathrm{L} \mathrm{NaHCO}_{3}$. SKCO15 cells stably expressing SNAP (New England BioLabs, Ipswich, MA) or SNAPFPR1 were transfected with pSNAP or pSNAP-FPR1 and selected with G418 $(1 \mu \mathrm{g} / \mathrm{mL})$.

\section{Antibodies, Reagents, and Plasmids}

The following primary monoclonal and polyclonal antibodies were used: anti-SNAP-tag (New England BioLabs), antihuman WDR26 (Sigma-Aldrich, St. Louis, MO), anti-FLAG (M2; Sigma-Aldrich), anti-c-myc (9B11; Cell Signaling Technology, Danvers, MA), anti-RhoA (1B12; Abcam, Cambridge, MA), anti-CDC42 (Becton, Dickinson and Co, Franklin Lakes, NJ), anti-Rac1 (Cell BioLabs, San Diego, CA), and anti-vav guanine nucleotide exchange factor (VAV)-2 (C64H2; Cell Signaling Technology). fMLP was purchased from Sigma-Aldrich. pSNAP-FPR1 and pcDNA3-c$m y c-$ FPR 1 were generated by subcloning of FPR1 amplified by high fidelity PCR using specific primers and subcloned into the NheI and EcoRI sites of pSNAPf (New England BioLabs) or XbaI and EcoRI sites of pcDNA3-c-myc, respectively. pcDNA3-FLAG-WDR26, pcDNA3-FLAG-WDR26 (1 to 122), pcDNA3-FLAG-WDR26 (1 to 274), pcDNA3-FLAGWDR26 (123 to 661), and pcDNA3-FLAG-WDR26 (275 to 661) were generated by subcloning of WDR26 amplified by PCR using specific primers and subcloned into the $\mathrm{XbaI}$ and ApaI sites of pcDNA3-FLAG.

\section{Transfection and Adenovirus Transduction}

Transient transfection of HEK293T cells, SKCO15 IECs, or HT29/B6 IECs was performed using Lipofectamine 2000 or 3000 (Thermo Fisher Scientific, Waltham, MA) in accordance with the manufacturer's instructions. WDR26 siRNA used for transient knockdown of WDR26 and control nontarget scramble siRNA were purchased from Horizon Discovery (Lafayette, CO). Transient transfections were performed using Lipofectamine RNAiMAX (Thermo Fisher Scientific) in accordance with the manufacturer's instructions. Fifteen minutes prior to adenoviral transduction, T84 IECs were subjected to calcium switch using a calciumchelating medium (10 mmol/L HEPES, $25 \mathrm{mmol} / \mathrm{L}$ EGTA, $\mathrm{pH}$ 7.4). After this, the FLAG-WDR26 and FLAG viruses were added at a multiplicity of infection of 100 . Cells were centrifuged for 1 hour at $1200 \times g$ at $25^{\circ} \mathrm{C}$ and then placed into a humidified incubator with $5 \% \mathrm{CO}_{2}$ at $37^{\circ} \mathrm{C}$. The cells were used 48 hours after initial virus addition.

\section{Wound-Healing Assays}

IECs were cultured on 24-well tissue culture plates to confluence and scratched using a $10-\mu \mathrm{L}$ pipette tip under suction as described previously. ${ }^{7}$ Video quantification of scratch wound healing was performed by the imaging of wounds at 1-hour intervals in an Axiovert Observer live cell microscopy system (Carl Zeiss Microscopy, White Plains, NY). Wound healing was quantified at the indicated time points using Photoshop software version 21.2 (Adobe Systems, San Jose, CA) and calculated as the percent reduction of cell-free surface area compared to immediately after wounding $(t=0)$.

\section{Pull-Down Assay and Immunoprecipitation Assay}

Cells were lysed in $10 \mathrm{mmol} / \mathrm{L}$ HEPES (pH 7.4), $100 \mathrm{mmol} /$ $\mathrm{L} \mathrm{KCl}, 10 \mathrm{mmol} / \mathrm{L} \mathrm{NaCl}, 1 \mathrm{mmol} / \mathrm{L}$ dithiothreitol, $2 \mathrm{mmol} / \mathrm{L}$ $\mathrm{MgCl}_{2}, 0.5 \% n$-dodecyl- $\beta$-D-maltoside containing protease and phosphatase inhibitor cocktails (Sigma-Aldrich). Pulldown assay was performed using SNAP-Capture PullDown Resin (New England BioLabs). Immunoprecipitation assay was performed using anti-c-myc antibody. Samples were then reduced and alkylated as described previously. ${ }^{20}$ Briefly, samples were incubated with $9 \mathrm{mmol} / \mathrm{L}$ dithiothreitol, $0.1 \mathrm{~mol} / \mathrm{L}$ Tris- $\mathrm{HCl}(\mathrm{pH} 8)$, and $1 \% \mathrm{SDS}$ at $60^{\circ} \mathrm{C}$ for 5 minutes and then cooled for 5 minutes at room temperature. The samples were further incubated with 
$60 \mathrm{mmol} / \mathrm{L} N$-ethylmaleimide at room temperature for 10 minutes and added to SDS sample buffer.

\section{Western Blot Analysis}

Samples were boiled for 5 minutes at $100^{\circ} \mathrm{C}$ in SDS sample buffer. Equal quantities of protein samples were loaded into $8 \%$ to $15 \%$ polyacrylamide gels for SDS-PAGE and transferred to polyvinylidene difluoride membrane. Membranes were blocked with $3 \%$ bovine serum albumin/Tris-buffered saline/ $0.1 \%$ Tween 20 , followed by overnight incubation with primary antibodies at $4{ }^{\circ} \mathrm{C}$. Membranes were then incubated with appropriate horseradish peroxidase-conjugated secondary antibodies for 1 hour at room temperature, followed by development using Clarity Western enhanced chemiluminescence substrate (Bio-Rad Laboratories, Hercules, CA), and image capture using the ChemiDoc imager (Cell BioLabs).

\section{RhoGTPase Assay}

Ras homolog family member A (RhoA), CDC42, and Rac1 activity assays were performed in accordance with the manufacturer's instructions (Cytoskeleton, Denver, CO). Briefly, transiently transfected cells were wounded and incubated with or without $1 \mu \mathrm{mol} / \mathrm{L}$ fMLP for 30 minutes and then lysed in kit-supplied buffer. Cell lysates were incubated with Rhotekin-Rho-binding domain (for RhoA) or p21-activated kinase-p21-activated kinase binding domain (for CDC42/ Rac1) agarose beads for 1 hour at $4^{\circ} \mathrm{C}$. The beads were washed and boiled in SDS sample buffer. Entire samples were then analyzed by Western blot analysis with detection by RhoA, CDC42, or Rac1 antibodies.

\section{Intracellular ROS Generation Assay}

ROS production was visualized by using redox-sensitive dye, CellROX oxidative stress reagents (Thermo Fisher Scientific). Transiently transfected cells treated with or without fMLP for 30 minutes were incubated with $5 \mu \mathrm{mol} / \mathrm{L}$ CellROX reagent for 30 minutes at $37^{\circ} \mathrm{C}$. ROS was detected by fluorescence microscopy. Quantification of fluorescence intensity of ROS was determined using ImageJ software version 1.51.23 (NIH, Bethesda, MD; https://imagej.nih. gov/ij).

\section{EdU Incorporation Assay}

Transiently transfected cells were incubated with $10 \mu \mathrm{mol} / \mathrm{L}$ 5-ethynyl-2'-deoxyuridine (EdU) for 1 hour. The amount of EdU incorporated into cells and nuclei (DAPI) was detected by immunofluorescence staining by using Click-it EdU imaging kits (Thermo Fisher Scientific) in accordance with the manufacturer's instructions. The percentage of cells positive for EdU over total cell nuclei (DAPI) was quantified using ImageJ software.

\section{Cell Migration Assay}

Cells were washed with phosphate-buffered saline and resuspended in serum-free media. A total of $1 \times 10^{5}$ cells were added on the top side of $12-\mu \mathrm{m}$ pore size, 24-well Transwell (Corning, Tewksbury, MA) inserts. Complete media was added into the bottom of the lower chamber in the plate. Cells were allowed to migrate across inserts toward the bottom side for 24 hours at $37^{\circ} \mathrm{C}$. Inserts were then washed, fixed with $70 \%$ ethanol, and stained with $0.2 \%$ crystal violet.

\section{Statistics}

Statistical significance was measured by one-way analysis of variance with the Tukey multiple-comparisons post-test using the Prism 8 software package (GraphPad Software, San Diego, CA). $P$ values of $<0.05$ were considered significant. Results are expressed as means \pm SEM.

\section{Results}

\section{WDR26 Interaction with FPR1 in IECs}

The ligation and activation of FPR1 promotes epithelial migration and wound repair. ${ }^{7,19}$ To further elucidate mechanisms of FPR1-mediated wound healing in IECs, FPR1-interacting proteins were investigated. SNAP-FPR1 or control SNAP-tag were stably expressed in SKCO15 IECs. SNAP proteins were pulled down with SNAP binding resin, and mass spectrometry was performed to identify SNAP-FPR1-associated proteins. These analyses identified FPR1 association with the WD40 repeat scaffold protein WDR26. To verify the association of FPR1 with WDR26 in a protein complex, the SNAP protein pull-down assay, followed by Western blot analysis using anti-WDR26 antibody (Figure 1A), was performed. WDR26 was detected in the FPR1-bound fraction in SNAP-FPR1 but not control SNAP fractions supporting WDR26 interaction with FPR1 in a protein complex.

\section{WDR26 Inhibition of FPR1-Mediated Cell Migration and Wound Healing in IECs}

Since FPR1 promotes epithelial cell migration and wound healing, it was tested whether WDR26-FPR1 interactions modulate FPR1-mediated wound healing. WDR26 was overexpressed in HT29/B6 IECs, and cell monolayers were subjected to scratch wound-healing assays. Wound healing was analyzed by time-lapsed imaging (Figure 1B). As shown in Figure 1B, fMLP stimulation enhanced wound healing in control FLAG-tag-expressed cells (nonstimulation versus fMLP stimulation at 12 hours, $42.2 \% \pm 2.5 \%$ versus $55.4 \% \pm 3.4 \%$ ). However, increased wound repair in response to fMLP stimulation was not observed in cells that overexpressed FLAG-WDR26 (nonstimulation versus fMLP stimulation at 12 hours, $40.6 \% \pm 2.5 \%$ versus $43.8 \% \pm 2.5 \%$ ). 

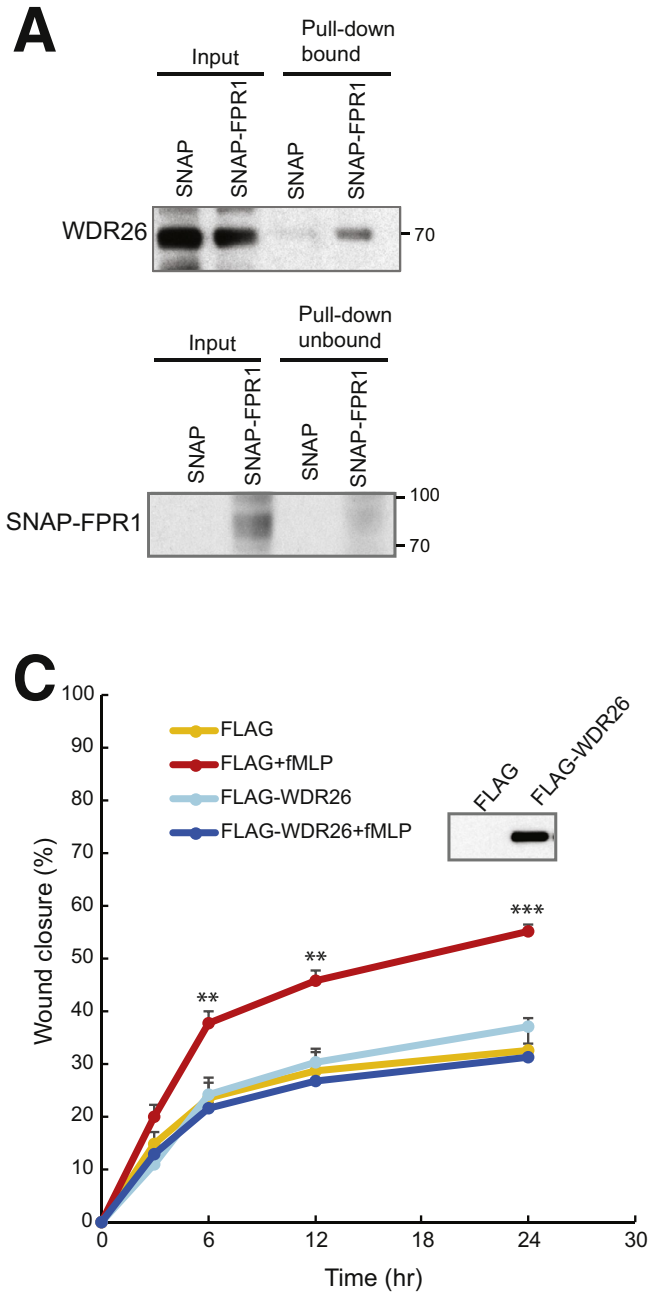
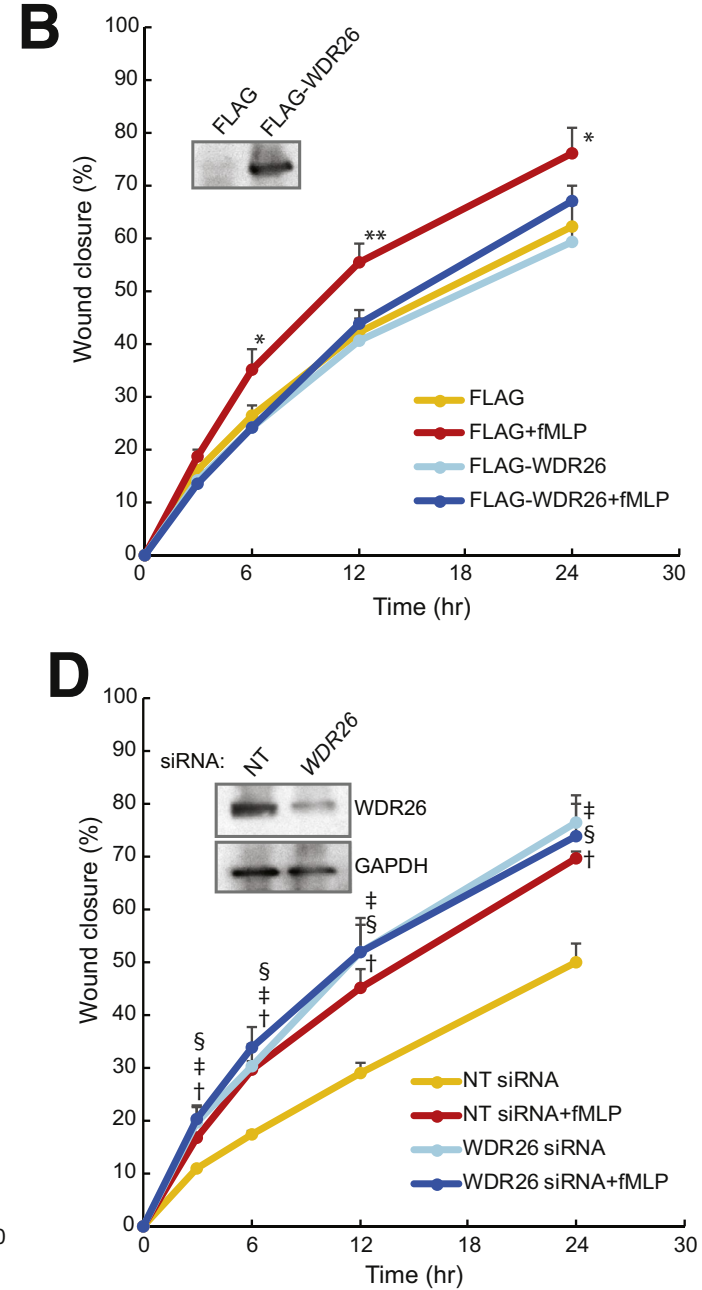

Figure 1 WD40 repeat protein 26 (WDR26) inhibits formyl peptide receptor 1 (FPR1)-mediated wound healing by interacting with FPR1. A: SNAP-FPR1 or SNAP (control) were stably expressed in SKCO15 cells. Cell lysates were subjected to pull-down assay using SNAP binding resin and separated into bound fraction and unbound fraction. The expression levels of WDR26 (top) and SNAP-FPR1 (bottom) are detected by Western blot analysis using anti-WDR26 and anti-SNAP antibodies, respectively. B: HT29/B6 cells were transfected with FLAG-WDR26 or FLAG (control). The expression of FLAG-WDR26 is confirmed by Western blot analysis using anti-FLAG antibodies (inset). Single linear wounds were created through confluent cell monolayers. The monolayers were treated with or without N-formyl-methionyl-leucyl phenylalanine (fMLP) $(1 \mu \mathrm{mol} / \mathrm{L})$, and wound healing was monitored for 24 hours. Differences between FLAG and FLAG-fMLP are statistically significant. C: T84 cells were infected with FLAG-WDR26 of FLAG (control) adenovirus. The expression of FLAG-WDR26 is confirmed by Western blot analysis using anti-FLAG antibodies (inset). Wound healing was monitored as in B. The differences between FLAG versus FLAG-fMLP are statistically significant. D: HT29/B6 cells were transfected with WDR26-specific siRNA or a nontargeting scramble siRNA (NT). Knockdown of WDR26 expression is confirmed by Western blot analysis using anti-WDR26 and anti-glyceraldehyde 3-phosphate dehydrogenase (GAPDH) antibodies as loading control (inset). Wound healing was monitored as in B. The differences between NT siRNA and NT siRNA + FMLP, NT siRNA and WDR26 siRNA, and NT siRNA and WDR26 SiRNA + fMLP are statistically significant. Data are expressed as means \pm SEM from three independent experiments. $n=6$ technical replicates per group. ${ }^{*} P<0.05,{ }^{* *} P<0.01$, and ${ }^{* * *} P<0.001$ versus FLAG-fMLP; ${ }^{\dagger} P<0.05$ versus NT siRNA + fMLP; ${ }^{\ddagger} P<0.05$ versus WDR26 siRNA; ${ }^{\S} P<0.05$ versus $W D R 26$ siRNA + fMLP (one-way analysis of variance).

Furthermore, analogous result of WDR26 overexpression on wound healing was observed in another model intestinal epithelial cell line, T84 (Figure 1C). fMLP stimulation enhanced wound healing in control FLAG-tag-transduced cells (nonstimulation versus fMLP stimulation at 12 hours, $28.8 \% \pm 3.9 \%$ versus $45.9 \% \pm 1.9 \%$ ). However, fMLP did not promote wound repair in cells that were FLAGWDR26-transduced (nonstimulation versus fMLP stimulation at 12 hours, $30.1 \% \pm 1.9 \%$ versus $26.8 \% \pm 2.3 \%$ ). Next, the effect of WDR26 on wound healing using WDR26knockdown cells (Figure 1D) was tested. Downregulation of
WDR26 protein by WDR26 siRNA enhanced wound healing compared with control nontarget scramble siRNA-transfected cells without fMLP stimulation (WDR26 siRNA versus nontarget siRNA at 12 hours, $51.7 \% \pm 5.3 \%$ versus $29.1 \% \pm 1.8 \%$ ). These results suggest that WDR26 negatively regulates epithelial FPR1-mediated wound repair.

A database search of conserved protein domains revealed that, in addition to the WD40 repeats, WDR26 also contains Lis-homology (LisH) and C-terminal to LisH (CTLH) domains at its $\mathrm{N}$-terminal segment (SMART; http://smart. embl-heidelberg.de, last accessed June 5, 2020) 
A

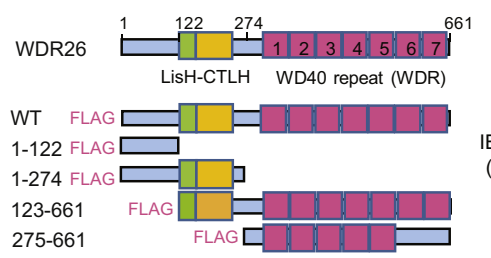

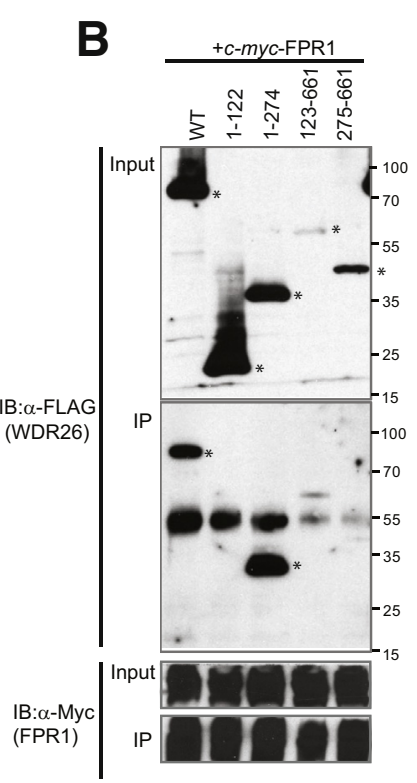

C

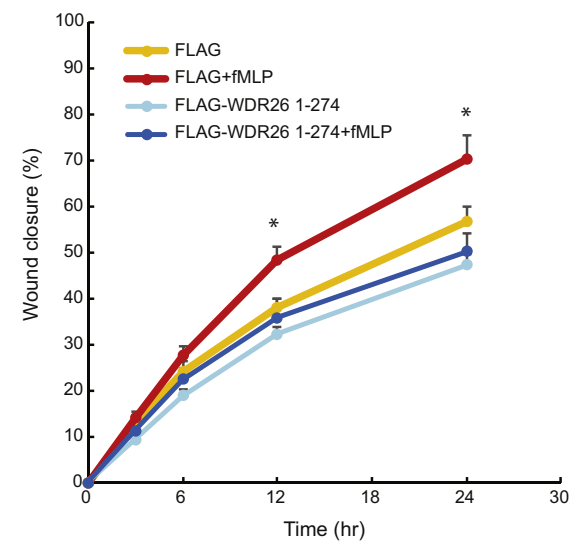

D

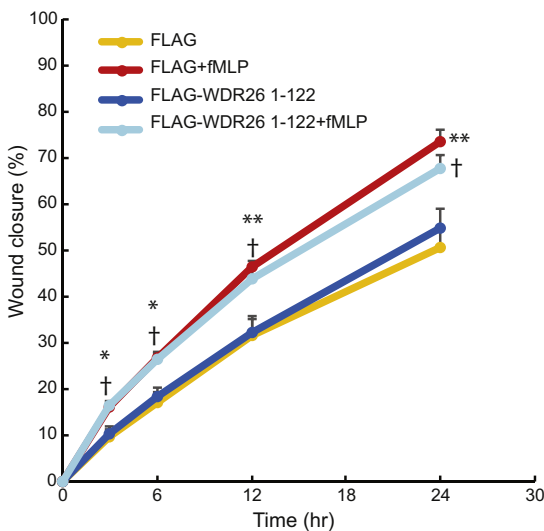

E

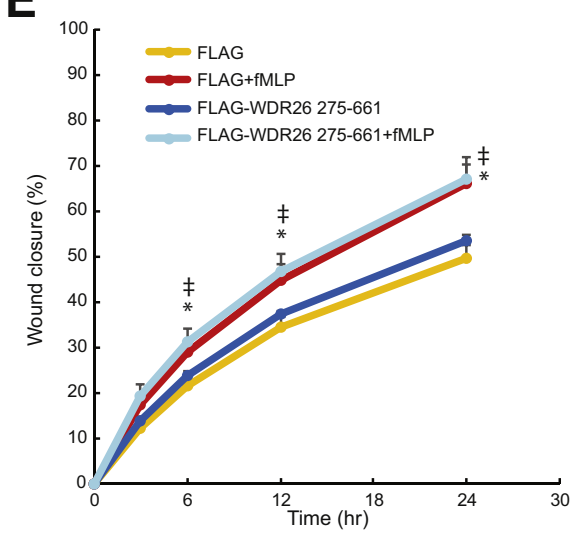

Figure 2 WD40 repeat protein 26 (WDR26) Lis-homology (LisH)-C-terminal to LisH (CTLH) domain interacts with formyl peptide receptor 1 (FPR1) and inhibits FPR1-mediated wound healing. A: WDR26 structure and the deletion mutants. B: HEK293T cells were co-transfected with c-myc-FPR1 and the indicated FLAG-WDR26 deletion mutant constructs. FPR1 was immunoprecipitated with anti-c-myc antibody. FPR1 and WDR26 were detected by Western blot analysis using anti-c-myc and anti-FLAG antibodies, respectively. Asterisks indicate bands at expected size for WDR26 full-length and deletion mutants. C-E: HT29/B6 cells were transfected with FLAG-WDR26 1-274 (C), FLAG-WDR26 1-122 (D), FLAG-WDR26 275-661 (E), or FLAG (control). Single linear wounds were created through confluent HT29/B6 cell monolayers. The monolayers were treated with or without N-formyl-methionyl-leucyl phenylalanine (fMLP) (1 $\mu$ mol/L), and wound healing was monitored for 24 hours. The differences between FLAG and FLAG + FMLP, FLAG-WDR26 1-122 and FLAG-WDR26 1-122 + fMLP, and FLAG-WDR26 275-661 and FLAG-WDR26 275-661 + fMLP are statistically significant. Data are expressed as means \pm SEM from three independent experiments. $n=6$ technical replicates per group. ${ }^{*} P<0.05,{ }^{*} P<0.01$ versus FLAG $+\mathrm{fMLP} ;{ }^{\dagger} P<0.05$ versus FLAG-WDR26 $1-122+\mathrm{fMLP} ;{ }^{\ddagger} P<0.05$ versus FLAG-WDR26 275-661 + fMLP (one-way analysis of variance). IB, immunoblot; IP, immunoprecipitation; WT, wild type.

(Figure 2A). To identify FPR1-binding sites on WDR26, a series of deletion mutants conjugated with FLAG, which were co-expressed with c-myc-FPR1 in HEK293T cells (Figure 2B) were generated. Of note, HEK293T cells were used because they were amenable to overexpression of WDR26 mutant proteins. Co-immunoprecipitation assays have indicated that full-length and 1-274 WDR26 interact with FPR1, suggesting that the binding sites on WDR26 are located at the N-terminus of WDR26 containing the LisHCTLH domain (Figure 2B). Since the N-terminus of WDR26 (1 to 274) interacts with FPR1, the effects of the WDR26 1-274 mutant on FPR1-mediated wound healing
(Figure 2C) was tested. Overexpression of WDR26 1-274 inhibited fMLP-induced wound healing to an extent similar to that of full-length WDR26 (Figure 1B). In contrast, WDR26 1-122 and 275-661 overexpressing cells maintained the ability to increase wound healing in response to fMLP (Figure 2, D and E). These results suggest that the WDR26 LisH-CTLH domain negatively regulates FPR1mediated wound healing.

Next, FPR1-mediated cell migration using a Boyden chamber was analyzed. WDR26-knockdown cells had enhanced migration even without fMLP stimulation, suggesting that WDR26 inhibits IEC migration (Supplemental 

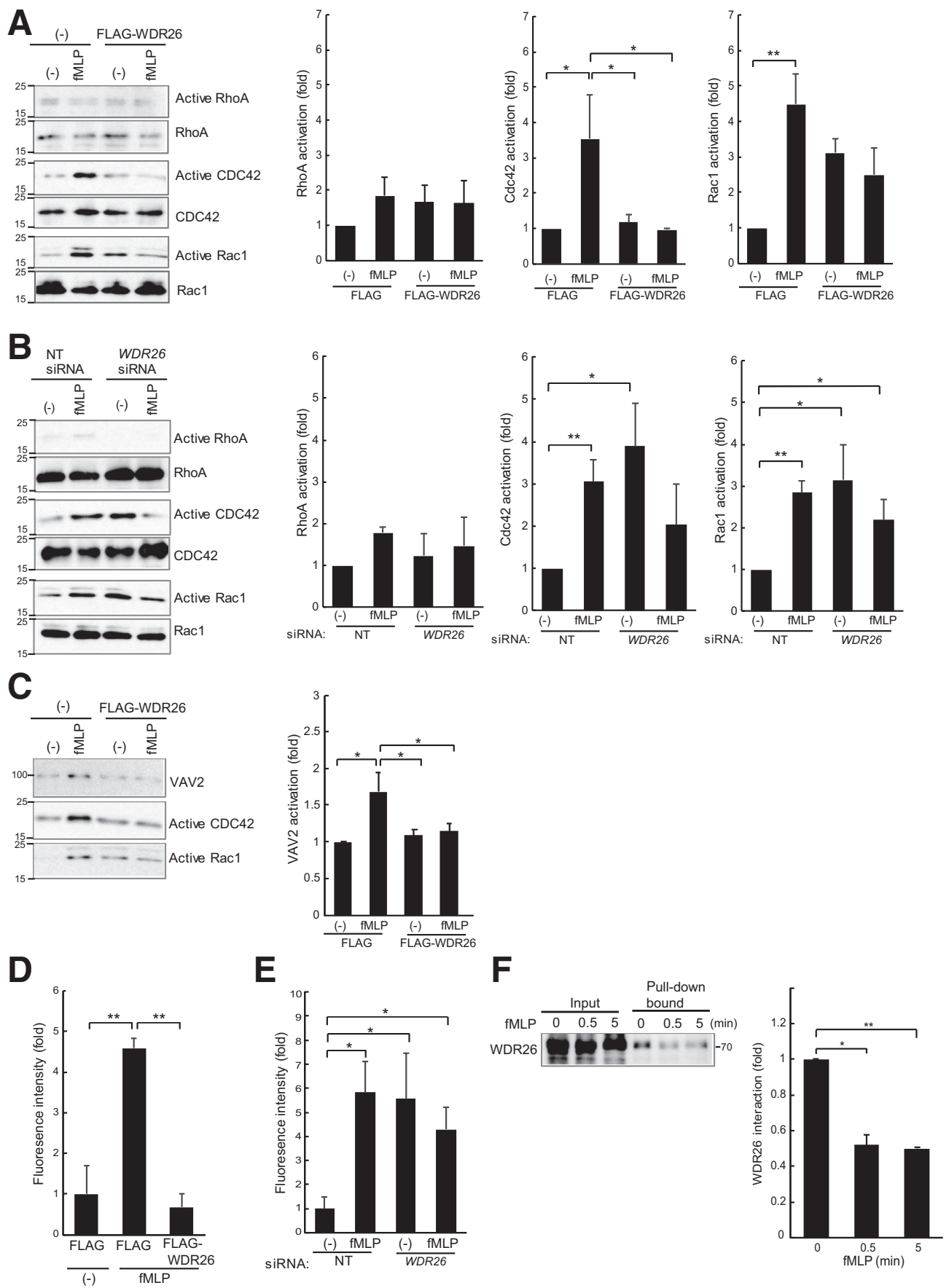

Figure 3 WD40 repeat protein 26 (WDR26) inhibits formyl peptide receptor 1 (FPR1)-mediated cell division cycle 42 (CDC42) and Rac family small GTPase 1 (Rac1) activation. A and B: HT29/B6 cells transfected with FLAG-WDR26 or FLAG (control) (A) and HT29/B6 cells transfected with WDR26-specific siRNA or with nontargeting scramble siRNA (NT) (B). Cell monolayers were wounded, and analysis of RhoGTPase activation was performed at 6 hours after injury. Wounded cell monolayers were untreated or treated with $\mathrm{N}$-formyl-methionyl-leucyl phenylalanine (fMLP) $(1 \mu \mathrm{mol} / \mathrm{L})$ for 30 minutes before cells were harvested. Active ras homolog family member A (RhoA) was pulled down using Rhotekin-Rho binding domain protein, whereas active CDC42 and active Rac1 were pulled down using p21-activated kinase-Rac/CDC42 (p21) binding domain. Total and active RhoGTPase were analyzed by Western blot analysis using anti-RhoA, anti-CDC42, and anti-Rac1 antibodies. Bar graphs show densitometric analysis of Western blots from three independent experiments. C: HT29/B6 cells were transfected with FLAG-WDR26 or FLAG (control). Cell monolayers were wounded and epithelial restitution was allowed for 6 hours. Wounded cell monolayers were untreated or treated with fMLP $(1 \mu \mathrm{mol} / \mathrm{L}) 30$ minutes before cells were harvested for analysis of RhoGTPase activation. Active CDC42 and Rac1 pull-down samples were subjected to Western blot analysis using anti-vav guanine nucleotide exchange factor (VAV)-2, antiCDC42, and anti-Rac1 antibodies. Bar graphs show densitometric analysis of Western blots for VAV2. D and E: HT29/B6 cells transfected with FLAG-WDR26 or FLAG (control) (D) and HT29/B6 cells transfected with WDR26-specific siRNA or nontargeting scramble siRNA (NT) (E). Cells were stimulated with or without fMLP (1 $\mu$ mol/L) for 30 minutes and then incubated with redox-sensitive dye, Hydro $\mathrm{Cy} 3$, to detect reactive oxygen species production. The fluorescence intensity of the probe Hydro Cy3 was analyzed by fluorescence microscopy. Bar graphs show fold-increase in fluorescence intensity compared to that in control cells. F: SNAP-FPR1 construct is stably expressed in SKC015 cells. Cells were untreated or treated with fMLP for 30 seconds or 5 minutes. SNAP-FPR1 was pulled down with SNAP binding resin, and WDR26 was detected by Western blot analysis using anti-WDR26 antibody. Data are expressed as means \pm SEM from three independent experiments. ${ }^{*} P<0.05,{ }^{* *} P<0.01$ (one-way analysis of variance). 

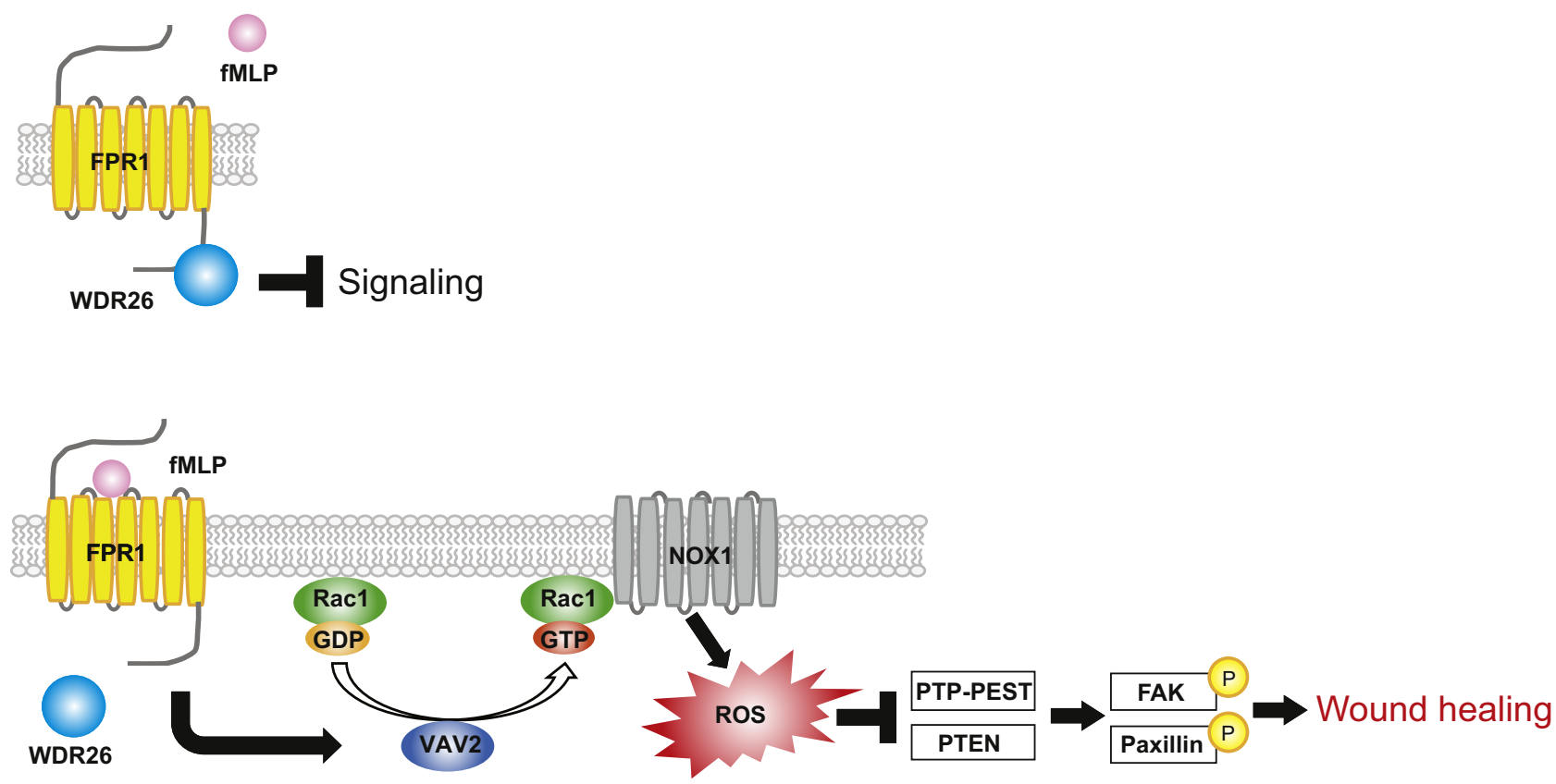

Figure 4 Mechanism by which formyl peptide receptor 1 (FPR1)-mediated wound healing is negatively regulated by WD40 repeat protein 26 (WDR26). Without stimulation, WDR26 interacts with FPR1 and inhibits FPR1-dependent signaling pathways. When FPR1 is activated by ligands such as N-formyl-methionyl-leucyl phenylalanine (FMLP), WDR26 is released from FPR1. Consequently, vav guanine nucleotide exchange factor (VAV)-2 associates with cell division cycle 42 (CDC42) and Rac family small GTPase 1 (Rac1), resulting in activation of these proteins and NADPH oxidase (NOX)-1-mediated intracellular reactive oxygen species (ROS) generation. ROS-mediated oxidative inactivation of select regulatory phosphatases is associated with phosphorylation and activation of proteins that promote cell movement and wound repair. FAK, focal adhesion kinase; PTEN, phosphatase and tensin homologue; PTP-PEST, protein tyrosine phosphatase with proline, glutamic acid, serine, and threonine residues.

Figure S1A). Since mucosal wound healing requires both epithelial migration and proliferation, cell proliferation by EdU incorporation assay was also analyzed. While fMLP treatment did not enhance epithelial proliferation rates, baseline proliferation was inhibited by WDR26 siRNA, supporting a role of WDR26 in promoting epithelial proliferation during wound repair (Supplemental Figure S1B). These results suggest that WDR26 inhibits cell migration and wound healing.

\section{WDR26 Inhibition of FPR1-Mediated CDC42 and Rac1 Activation in IECs}

Since Rho GTPases are known to play a central role in regulating cellular functions involved in cell migration, it was tested whether WDR26 regulates RhoGTPase activation in epithelial cells. fMLP-induced activation of RhoA, CDC42, and Rac1 was tested by respective effector binding assays (Rhotekin-agarose or p21-activated kinase agarose). fMLP stimulation activated CDC42 and Rac1 but not RhoA in control cells expressing the FLAG construct. However, the expression of FLAG-WDR26 inhibited the activation of CDC42 and Rac1 (Figure 3A). Furthermore, CDC42 and Rac1 were activated even without fMLP stimulation in WDR26-knockdown cells (Figure 3B). Taken together, these data suggest that WDR26 negatively regulates CDC42 and Rac1 activation during FPR1 signaling.

The activation of CDC42 and Rac1 is catalyzed by guanine nucleotide exchange factors. FPR1 activation has previously been shown to increase the association between guanine nucleotide exchange factors VAV2 and active Rac1 complex. ${ }^{19}$ Therefore, it was tested whether VAV2 associates with active Rac1 and CDC42 after fMLP stimulation of IECs. Pull-down using p21-activated kinase agarose identified VAV2 in a complex with active CDC42 and Rac1. It was found that fMLP stimulation increased the association of VAV2 with active CDC42 and Rac1. This association was inhibited in cells overexpressing WDR26 (Figure 3C), suggesting that WDR26 negatively regulates the association of VAV2 with active CDC42/Rac1 in fMLP-stimulated IECs.

Rac1 is a known regulator of NOX1, which induces intracellular ROS generation and oxidative inactivation of protein tyrosine phosphatase with proline, glutamic acid, serine, and threonine residues (PTP-PEST) and phosphatase and tensin homologue (PTEN), resulting in activation of focal adhesion proteins. ${ }^{19}$ Therefore, it was tested whether FPR1-mediated ROS generation is regulated by WDR26. fMLP stimulation resulted in ROS generation in control IECs expressing the FLAG-tag. However, this increase in fMLP-induced ROS generation was abrogated in IECs expressing FLAG-WDR26 (Figure 3D). In contrast, increased ROS generation in IECs was observed after WDR26 knockdown with or without fMLP stimulation (Figure 3E). These results suggest that WDR26 negatively regulates FPR1-mediated ROS generation.

\section{WDR26 Dissociation from FPR1 after fMLP Stimulation}

Since WDR26 interacts with FPR1 and inhibits downstream FPR1 signaling, it was hypothesized that in the absence of 
exogenous stimuli, WDR26 interacts with FPR1 to inhibit downstream signaling. The data from the present study suggest that fMLP stimulation releases WDR26 from the FPR1 protein complex and facilitates the activation of FPR1 signaling and epithelial cell migration. Therefore, changes in the physical association of FRP1 and WDR26 after fMLP stimulation were examined. As shown in Figure 3F, decreased WDR26 was detected in FPR1 pull-down assays. This result is consistent with the association of WDR26 with FPR1 in a protein complex that is lost after fMLP stimulation.

\section{Discussion}

Formylated peptides generated from luminal bacteria and proteins released from injured cells are potent agonists of FPRs, and lead to the recruitment and activation of leukocytes at sites of mucosal injury. ${ }^{3-5}$ In addition to the regulation of leukocyte function, the activation of epithelialdependent FPR signaling promotes efficient epithelial wound repair after injury. ${ }^{7,19}$ In the terminal phase of wound repair, epithelial cell migration is inhibited so as to restore the epithelial barrier. Mechanisms that orchestrate such terminal healing events are, however, not well understood. This study identified WDR26 as a novel FPR1-interacting protein in IECs. We demonstrate that WDR26 negatively regulates FPR1 signaling and epithelial repair by inhibiting the activation of the small GTPases Rac1 and CDC42 in response to fMLP-mediated activation of FPR1.

Previous studies have linked WDR26 to Wnt and mitogen-activated protein kinase inhibition, phospholipase $\mathrm{C} \beta 2$ activation, the phosphatidylinositol 3 kinase-AKT axis, ubiquitination processes, protection against oxidative stress, Rac1 translocation, $\mathrm{G} \beta / \gamma$ subunit-triggered chemotaxis, and cell migration. ${ }^{21-29}$ However, the regulation of FPR1 activity by WDR26 has not been previously identified.

WDR26 has WD40 repeat domains that regulate its interaction with a number of proteins, thereby functioning as a scaffold protein. ${ }^{30-32}$ However, this study demonstrated that WDR26 associated with FPR1 through its LisH-CTLH domain. Therefore, we believe that the WD40 repeat domain is not required for its interaction with FPR1 and consequent downstream signaling. CTLH proteins have previously been linked to fundamental biological processes, including proliferation, survival, regulated cell death, cell adhesion, cell migration, and DNA-damage responses in oncogenic signaling. ${ }^{33}$ These findings have revealed a novel function for the LisH-CTLH domain of WDR26 in modulating epithelial repair.

The activation of FPR1 in IECs results in dissociation of heterotrimeric G-proteins into $\alpha$ and $\beta \gamma$ subunits, with consequent activation of downstream signaling. WDR26 has been identified as a G $\beta$-like protein with a similar ternary structure. $^{22}$ It was observed that WDR26 interacted with FPR1 in the absence of fMLP stimulation. However, on exposure to fMLP, WDR26 association with FPR1 was decreased in IECs, suggesting that WDR26 functions as an on-off switch for FPR1 signaling in a manner similar to that observed downstream of receptors after their dissociation from $\mathrm{G}$ proteins. A previous report showed that WDR26 interacted and regulated $\mathrm{G} \beta \gamma$-mediated signaling in breast cancer cells through the promotion of cell growth, migration, and invasion. ${ }^{24}$ WDR26 has been shown to regulate the function of diverse G-protein-coupled receptors by interacting with $G \beta \gamma$, including lysophosphatidic acid receptors, chemokine receptors (eg, C-X-C motif chemokine receptors 4 and 7), and protease-activated receptors. ${ }^{24}$ Whereas FPR1 belongs to the G-protein-coupled receptor family, no interaction between WDR2 6 and G $\beta \gamma$ was detected, and WDR2 6 interacted directly with FPR1 through its LisH-CTLH domain in IECs. Furthermore, unlike a previous report, ${ }^{24}$ here it was observed that WDR26 inhibited IEC migration. All together, these findings suggest that WDR26 is a specific negative regulator of FPR1. Nonetheless, we cannot rule out that WDR26 can modulate the function of other FPRs as well as G-protein-coupled receptors in IECs, and this hypothesis requires further investigation.

Of note, FPR 1 is expressed in IECs as well as in immune cells. The activation of FPR1 in immune cells also induces its dissociation from heterotrimeric $G$ proteins ( $G \alpha$ and $G \beta \gamma)$, resulting in downstream signaling cascades that promote a number of host-defense and antimicrobial-immune responses. ${ }^{3-5}$ WDR26 is ubiquitously expressed; therefore, it is tempting to speculate that the WDR26-FPR1 interaction might occur in immune cells and contribute to the inhibition of FPR1 signaling in the absence of stimuli, thereby functioning to fine-tune the receptor function. However, this potential role of the WDR26-FPR1 complex in the regulation of the immune response requires further study.

These studies have shown that CDC42 and Rac1 were activated downstream of FPR1 activation. The Rho GTPases (RhoA, CDC42, and Rac1) are central regulators of F-actin restructuring, which is required for cell movement. ${ }^{34-37}$ The activation state of Rho GTPases is dependent on reversible interactions with triphosphorylated guanine nucleotides (GTP). Rho GTPase activation is directly regulated by guanine nucleotide exchange factors, exchange factors that catalyze the exchange of GDP to GTP. ${ }^{38}$ Consistent with a previous report, ${ }^{19}$ it was demonstrated that the guanine nucleotide exchange factors VAV2 associated with active CDC42 and Rac1 in IECs after fMLP stimulation. This result suggests that VAV2 catalyzes the exchange of GDP to GTP on CDC42 and Rac1 after FPR1 activation. This association was decreased by WDR26 overexpression, suggesting that WDR26 negatively controls VAV2 activity, which, in turn, reduces CDC42 and Rac1 activation.

FPRs are classically appreciated to promote chemotaxis and microbicidal oxidant burst in neutrophils. Neutrophil FPR activation with fMLP has been reported to induce NOX2 activation and result in high levels of ROS 
generation, which mediates host defense against pathogens. ${ }^{39}$ Here, a beneficial role of FPR signaling in mediating intracellular IEC ROS generation, which exerted a prorepair function is observed. The downstream beneficial effects of NOX1-dependent ROS signaling, in turn, are mediated by oxidative inactivation of key regulatory phosphatases that regulate actin cytoskeletal and focal adhesion dynamics. ${ }^{40}$ A previous report demonstrated that phosphatase PTEN and PTP-PEST were rapidly and transiently inactivated by ROS in cells treated with the FPR1 ligand Ac2-26. PTP-PEST and PTEN have been reported to directly regulate the tyrosine phosphorylation of the focal cell matrix adhesion proteins focal adhesion kinase and paired box protein Pax, thereby orchestrating cell motility. ${ }^{19}$

In concert with our current results, a model is proposed in which WDR26 interaction with FPR1 functions to inhibit this signaling cascade and wound repair in response to fMLP stimulation. After FPR1 activation with fMLP, WDR26 inhibition was released from FPR1, resulting in the activation of CDC42/Rac1 through the association of a guanine nucleotide exchange factor VAV2. CDC42/Rac1 was found to activate NOX1 and induce intracellular ROS generation, which control proteins in focal cell matrix adhesions to regulate cell motility (Figure 4). Additional studies are needed to determine how fMLP stimulation modulates the binding of WDR26 to regulate epithelial cell motility as well as to characterize the intrinsic physiologic functions of WDR26 in vivo in intestinal mucosal homeostasis and in disorders such as inflammation and cancer.

Taken together, our results demonstrate a crucial role of WDR26 in the regulation of FPR1-dependent signaling during the resolution of inflammation, promoting efficient repair of injured intestinal epithelium and restoration of mucosal homeostasis. Our findings suggest that targeting WDR26-FPR1 interaction could be a potential therapeutic approach to promoting wound repair and restoring intestinal epithelial barrier function.

\section{Acknowledgments}

We thank Dr. Roland Hilgarth for facilitating the experiments and Dr. Jennifer Brazil and Dr. Anny-Claude Luissint (University of Michigan) for manuscript review and discussion.

\section{Supplemental Data}

Supplemental material for this article can be found at https://doi.org/10.1016/j.ajpath.2020.06.005.

\section{References}

1. Takeuchi O, Akira S: Pattern recognition receptors and inflammation. Cell 2010, 140:805-820
2. Brubaker SW, Bonham KS, Zanoni I, Kagan JC: Innate immune pattern recognition: a cell biological perspective. Annu Rev Immunol 2015, 33:257-290

3. Weiß E, Kretschmer D: Formyl-peptide receptors in infection, inflammation, and cancer. Trends Immunol 2018, 39:815-829

4. Filep JG, Sekheri M, El Kebir D: Targeting formyl peptide receptors to facilitate the resolution of inflammation. Eur J Pharmacol 2018, 833:339-348

5. Raabe CA, Gröper J, Rescher U: Biased perspectives on formyl peptide receptors. Biochim Biophys Acta Mol Cell Res 2019, 1866: $305-316$

6. Babbin BA, Lee WY, Parkos CA, Winfree LM, Akyildiz A, Perretti M, Nusrat A: Annexin I regulates SKCO-15 cell invasion by signaling through formyl peptide receptors. J Biol Chem 2006, 281: 19588-19599

7. Babbin BA, Jesaitis AJ, Ivanov AI, Kelly D, Laukoetter M, Nava P, Parkos CA, Nusrat A: Formyl peptide receptor-1 activation enhances intestinal epithelial cell restitution through phosphatidylinositol 3kinase-dependent activation of Rac1 and Cdc42. J Immunol 2007, 179:8112-8121

8. Schiffmann E, Corcoran BA, Wahl SM: N-formylmethionyl peptides as chemoattractants for leucocytes. Proc Natl Acad Sci U S A 1975 , 72:1059-1062

9. Marasco WA, Phan SH, Krutzsch H, Showell HJ, Feltner DE, Nairn R, Becker EL, Ward PA: Purification and identification of formyl-methionyl-leucyl-phenylalanine as the major peptide neutrophil chemotactic factor produced by Escherichia coli. J Biol Chem 1984, 259:5430-5439

10. Brazil JC, Quiros M, Nusrat A, Parkos CA: Innate immune cellepithelial crosstalk during wound repair. J Clin Invest 2019, 129: 2983-2993

11. Neurath MF, Travis SP: Mucosal healing in inflammatory bowel diseases: a systematic review. Gut 2012, 61:1619-1635

12. Papi C, Aratari A: Mucosal healing as a treatment for IBD? Expert Rev Gastroenterol Hepatol 2014, 8:457-459

13. Quirós M, Nusrat A: Contribution of wound-associated cells and mediators in orchestrating gastrointestinal mucosal wound repair. Annu Rev Physiol 2019, 81:189-209

14. Wentworth CC, Jones RM, Kwon YM, Nusrat A, Neish AS: Commensal-epithelial signaling mediated via formyl peptide receptors. Am J Pathol 2010, 177:2782-2790

15. Wentworth CC, Alam A, Jones RM, Nusrat A, Neish AS: Enteric commensal bacteria induce extracellular signal-regulated kinase pathway signaling via formyl peptide receptor-dependent redox modulation of dual specific phosphatase 3. J Biol Chem 2011, 286: $38448-38455$

16. Kumar A, Wu H, Collier-Hyams LS, Kwon YM, Hanson JM, Neish AS: The bacterial fermentation product butyrate influences epithelial signaling via reactive oxygen species-mediated changes in cullin-1 neddylation. J Immunol 2009, 182:538-546

17. Swanson PA 2nd, Kumar A, Samarin S, Vijay-Kumar M, Kundu K, Murthy N, Hansen J, Nusrat A, Neish AS: Enteric commensal bacteria potentiate epithelial restitution via reactive oxygen speciesmediated inactivation of focal adhesion kinase phosphatases. Proc Natl Acad Sci U S A 2011, 108:8803-8808

18. Alam A, Leoni G, Wentworth CC, Kwal JM, Wu H, Ardita CS, Swanson PA, Lambeth JD, Jones RM, Nusrat A, Neish AS: Redox signaling regulates commensal-mediated mucosal homeostasis and restitution and requires formyl peptide receptor 1. Mucosal Immunol 2014, 7:645-655

19. Leoni G, Alam A, Neumann PA, Lambeth JD, Cheng G, McCoy J, Hilgarth RS, Kundu K, Murthy N, Kusters D, Reutelingsperger C, Perretti M, Parkos CA, Neish AS, Nusrat A: Annexin A1, formyl peptide receptor, and NOX1 orchestrate epithelial repair. J Clin Invest 2013, 123:443-454

20. Maaty WS, Lord CI, Gripentrog JM, Riesselman M, Keren-Aviram G, Liu T, Dratz EA, Bothner B, Jesaitis AJ: Identification of C-terminal 
phosphorylation sites of $\mathrm{N}$-formyl peptide receptor-1 (FPR1) in human blood neutrophils. J Biol Chem 2013, 288:27042-27058

21. Goto T, Matsuzawa J, Iemura S, Natsume T, Shibuya H: WDR26 is a new partner of axin 1 in the canonical Wnt signaling pathway. FEBS Lett 2016, 590:1291-1303

22. Zhu Y, Wang Y, Xia C, Li D, Li Y, Zeng W, Yuan W, Liu H, Zhu C, Wu X, Liu M: WDR26: a novel Gbeta-like protein, suppresses MAPK signaling pathway. J Cell Biochem 2004, 93:579-587

23. Sun Z, Smrcka AV, Chen S: WDR26 functions as a scaffolding protein to promote $\mathrm{G} \beta \gamma$-mediated phospholipase C $\beta 2$ (PLC $\beta 2)$ activation in leukocytes. J Biol Chem 2013, 288:16715-16725

24. Ye Y, Tang X, Sun Z, Chen S: Upregulated WDR26 serves as a scaffold to coordinate PI3K/AKT pathway-driven breast cancer cell growth, migration, and invasion. Oncotarget 2016, 7:17854-17869

25. Maitland MER, Onea G, Chiasson CA, Wang X, Ma J, Moor SE, Barber KR, Lajoie GA, Shaw GS, Schild-Poulter C: The mammalian CTLH complex is an E3 ubiquitin ligase that targets its subunit muskelin for degradation. Sci Rep 2019, 9:9864

26. Francis O, Han F, Adams JC: Molecular phylogeny of a RING E3 ubiquitin ligase, conserved in eukaryotic cells and dominated by homologous components, the muskelin/RanBPM/CTLH complex. PLoS One 2013, 8:e75217

27. Zhao J, Liu Y, Wei X, Yuan C, Yuan X, Xiao X: A novel WD-40 repeat protein WDR26 suppresses H2O2-induced cell death in neural cells. Neurosci Lett 2009, 460:66-71

28. Feng Y, Zhang C, Luo Q, Wei X, Jiang B, Zhu H, Zhang L, Jiang L, Liu M, Xiao X: A novel WD-repeat protein, WDR26, inhibits apoptosis of cardiomyocytes induced by oxidative stress. Free Radic Res 2012, 46:777-784
29. Castro-Castro A, Muriel O, Del Pozo MA, Bustelo XR: Characterization of novel molecular mechanisms favoring Rac1 membrane translocation. PLoS One 2016, 11:e0166715

30. Jain BP, Pandey S: WD40 repeat proteins: signalling scaffold with diverse functions. Protein J 2018, 37:391-406

31. Schapira M, Tyers M, Torrent M, Arrowsmith CH: WD40 repeat domain proteins: a novel target class? Nat Rev Drug Discov 2017, 16:773-786

32. Stirnimann CU, Petsalaki E, Russell RB, Müller CW: WD40 proteins propel cellular networks. Trends Biochem Sci 2010, 35:565-574

33. Huffman N, Palmieri D, Coppola V: The CTLH complex in cancer cell plasticity. J Oncol 2019, 2019:4216750

34. Fenteany G, Janmey PA, Stossel TP: Signaling pathways and cell mechanics involved in wound closure by epithelial cell sheets. Curr Biol 2000, 10:831-838

35. Nobes CD, Hall A: Biochem. Rho, rac and cdc 42 GTPases: regulators of actin structures, cell adhesion and motility. Soc Trans 1995, 23:456-459

36. Nobes CD, Hall A: Rho GTPases control polarity, protrusion, and adhesion during cell movement. J Cell Biol 1999, 144:1235-1244

37. Santos MF, McCormack SA, Guo Z, Okolicany J, Zheng Y, Johnson LR, Tigyi G: Rho proteins play a critical role in cell migration during the early phase of mucosal restitution. J Clin Invest 1997, 100:216-225

38. Schmidt A, Hall A: Guanine nucleotide exchange factors for Rho GTPases: turning on the switch. Genes Dev 2002, 16:1587-1609

39. Bylund J, Samuelsson M, Collins LV, Karlsson A: NADPH-oxidase activation in murine neutrophils via formyl peptide receptors. Exp Cell Res 2003, 282:70-77

40. Hurd TR, DeGennaro M, Lehmann R: Redox regulation of cell migration and adhesion. Trends Cell Biol 2012, 22:107-115 